\title{
CONSISTENCY OF A RANK TEST AGAINST GENERAL ALTERNATIVES OF CHANGE POINTS (SURFACES) AND CONTINUOUS TREND
}

\author{
A. I. Katsevich, A. G. Ramm
}

\begin{abstract}
Considered are modifications of a rank test of randomness for the oneand multidimensional regular design cases as well as for the one- and multidimensional random design cases. The null hypothesis is that all observations are independent and identically distributed. The main result is the proof of consistency of the test in each of the above cases against two general alternatives. Alternative 1: there exists a pairwise disjoint partition $\cup_{i=1}^{m} D_{i}=D$, where $D \subset \mathbb{R}^{d}, d \geq 1$, is a bounded domain inside which one makes observations, such that (1) if an observation point falls inside $D_{i}$, then the corresponding observed value is the realization of a random variable $\xi_{i}, i=1, \ldots, m ;(2)$ there exists an ordering $\left\{\xi_{i_{k}}\right\}_{k=1}^{m}$, where $\xi_{i_{k}}$ is stochastically smaller than $\xi_{i_{k+1}}, k=1, \ldots, m-1$, (3) the partition is independent of the number of observation points. Note that $m$, this ordering, and the sets $D_{i}$ are not known a priori: one tests only for the existence of such a partition. Note also that in the one-dimensional case the initial sequence need not be stochastically monotone under the alternative. Alternative 2: there exists an arbitrary "asymptotically continuous" trend in location. "Asymptotically continuous" means that the trend converges to some continuous, not identically constant function as the number of data points goes to infinity. This function need not be monotone.

A numerical example illustrating the use of the obtained results for image analysis
\end{abstract} (edge detection) is presented.

\section{Introduction}

In this paper ${ }^{1}$ we consider modifications of a rank test of randomness for the one- and multidimensional regular design cases as well as for the one- and multidimensional random design cases. The null hypothesis is that all observations are independent and identically distributed. Our main result is the proof of consistency of the test in each of the above cases against two general alternatives.

Alternative 1. There exists a pairwise disjoint partition $\cup_{i=1}^{m} D_{i}=D$, where $D \subset$ $\mathbb{R}^{d}, d \geq 1$, is a bounded domain inside which one makes observations, such that

(1) if an observation point falls inside $D_{i}$, then the corresponding observed value is the realization of a random variable $\xi_{i}, i=1, \ldots, m$,

1991 Mathematics Subject Classification. Primary 62G10.

Key words and phrases. Rank test, test of randomness, change points, trend, consistency.

The authors thank Dr. V. Faber for support. This research was performed under the auspices of the U.S. Department of Energy

${ }^{1}$ This paper was published in Acta Applicandae Mathematicae Vol. 42, pp. 105-137, 1996 
(2) there exists an ordering $\left\{\xi_{i_{k}}\right\}_{k=1}^{m}$, where $\xi_{i_{k}}$ is stochastically smaller than $\xi_{i_{k+1}}, k=1, \ldots, m-1$

(3) the partition is independent of the number $N$ of observation points.

Alternative 2. There exists an arbitrary "asymptotically continuous" trend in location. In the regular design model, assumptions on the trend are the following. In the one-dimensional case the trend $\left\{\theta_{k}\right\}_{k=1}^{N}$ is supposed to satisfy the condition:

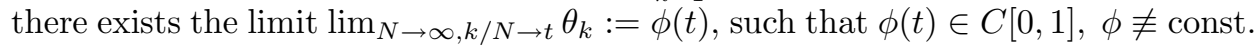
In the multidimensional case let the given data be $x_{k_{1}, \ldots, k_{d}}, 1 \leq k_{i} \leq \beta_{i} N, 0<$ $\beta_{i}<\infty, i=1, \ldots, d$. Denote $B_{N}:=\left\{k=\left(k_{1}, \ldots, k_{d}\right) \in \mathbb{N}^{d}: 1 \leq k_{i} \leq \beta_{i} N, i=\right.$ $1, \ldots, d\}, D:=\left\{t=\left(t_{1}, \ldots, t_{d}\right) \in \mathbb{R}^{d}: 0 \leq t_{i} \leq \beta_{i}, i=1, \ldots, d\right\}$. The condition on the trend $\left\{\theta_{k}, k \in B_{N}\right\}$ is: $\lim _{N \rightarrow \infty, k / N \rightarrow t} \theta_{k}:=\phi(t) \in C[D], \phi \not \equiv$ const. In the random design model, we assume that observation points $\left\{\rho_{k}\right\}_{k=1}^{N}$ are randomly chosen inside an open bounded domain $D \subset \mathbb{R}^{d}, d \geq 1$ (that is, $\left\{\rho_{k}\right\}_{k=1}^{N}$ are independent random points distributed in $D$ with constant probability density), and the trend at each point is given by $\theta_{k}=\phi\left(\rho_{k}\right)$, where $\phi(t) \in C(\bar{D}), \phi \not \equiv$ const, and $\bar{D}$ is the closure of $D$.

Note that under the first alternative, $m$, the ordering $\left\{\xi_{i_{k}}\right\}_{k=1}^{m}$, and the sets $D_{i}$ are not known a priori: one tests only for existence of such a partition. Thus Alternative 1 is different from the standard $m$-sample alternatives [M], where boundaries between samples are supposed to be known. In the one-dimensional case our alternative reduces to the existence of change points, and we do not assume that the initial sequence is stochastically monotone: there can be both jumps up and jumps down. Note also that in the case $m=2$ there can be any finite number of change points, because the sets $D_{1}$ and $D_{2}$ can be multiconnected.

In the one-dimensional case, equispaced design model, the statistic we use is

$$
\nu_{N}:=\frac{1}{N-1} \sum_{k=1}^{N-1}\left(\frac{R_{k+1}-R_{k}}{N}\right)^{2},
$$

where $R_{k}$ is the rank of the $k$-th element of the sequence to be tested, $k=1, \ldots, N$. We see that $\nu_{N}$ is closely related to the rank statistic $R$ introduced by Wald and Wolfowitz [WW]: $R=\sum_{i=1}^{N-1} R_{i} R_{i+1}+R_{1} R_{N}$. We have

$$
N^{2}(N-1) \nu_{N}=N(N+1)(2 N+1) / 3-2 R-\left(R_{1}-R_{N}\right)^{2}
$$

thus $\nu_{N}$ and $R$ are asymptotically equivalent. Many results are known concerning the statistic $R$ : the asymptotic normality, consistency against monotone trend, cyclical movement, serial correlation and some other alternatives [WW, N, A, AGA]. Also note that $\nu_{N}$ has the form of the Durbin-Watson statistic [DW] with observations replaced by their ranks. However, we could not find any proofs of consistency against the two general alternatives we consider. The most frequently considered alternatives are one change point, monotone trend and serial correlation $[\mathrm{B}, \mathrm{CH}$, $\mathrm{KO}, \mathrm{KM}, \mathrm{KS}]$. Different rank tests and different results for the case of multiple change points can be found in $[\mathrm{L}]$. Our results were announced in a brief form in [KR2]. In $[\mathrm{K}],[\mathrm{KR} 1]$, [KR3], and [KR4], related problems are discussed from a different point of view. 
In the multidimensional case, regular design model, the statistic we use is based on a modification of the Geary statistic $[\mathrm{G}, \mathrm{CO}]$ with observations replaced by their ranks:

$$
\nu_{N}:=\frac{1}{M_{N}} \sum_{k \in B_{N}} \sum_{l \in L(k)}\left(\frac{R_{k}-R_{l}}{\hat{N}}\right)^{2},
$$

where $L(k)$ is the set of lattice points neighboring to a point $k, M_{N}$ is the number of elements in double sum (1.3), and $\hat{N}$ is the number of lattice points.

In the case of the random design model, the analog of (1.1) and (1.3) is

$$
\nu_{N}:=\frac{1}{N} \sum_{k=1}^{N}\left(\frac{R_{n(k)}-R_{k}}{N}\right)^{2},
$$

where $\left\{\rho_{k}\right\}_{k=1}^{N}$ is a set of random observation points inside a bounded domain, and $n(k)$ is the index of the point closest to $\rho_{k}$ (the nearest neighbor).

The paper is organized as follows. In Section 2 we consider Alternative 1. In Sections 2.1 and 2.2 the proof of consistency in the one-dimensional cases: $m=2$ and $m>2$ is given. It is based on the following approach. First, we prove that $\nu_{N} \underset{N \rightarrow \infty}{\stackrel{\mathrm{ms}}{\longrightarrow}} 1 / 6$ under the hypothesis of randomness, and that $\nu_{N} \underset{N \rightarrow \infty}{\stackrel{\mathrm{ms}}{\longrightarrow}} E_{m}$ under the alternative. Here $E_{m}$ is some constant, and " $\underset{N \rightarrow \infty}{\mathrm{ms}}$ " denotes convergence in mean square. Since the asymptotic behavior of the Wald-Wolfowitz statistic $R$, and therefore that of $\nu_{N}$, under the hypothesis of randomness is well known [WW], we present values of the first two moments of $\nu_{N}$ without detailed calculations. Under the alternative, the behavior of $\nu_{N}$ has been studied less extensively, although convergence $\nu_{N} \underset{N \rightarrow \infty}{\stackrel{\mathrm{ms}}{\longrightarrow}} E_{m}$ can be easily established by a standard technique. Thus, we only sketch the proof of this fact in the case $m=2$. In the case $m>2$, it can be proved similarly, so we presented only the final formulas. The main new point is the proof of the inequality $E_{m}<1 / 6$, so it is given in detail. In Section 2.3 the case of the data specified at the nodes of a regular $d$-dimensional grid is considered. Since this case is completely analogous to the one-dimensional case, we only describe the model, statistic, and state main results without proofs. In Section 2.4 the case of data points randomly distributed inside a certain bounded domain is considered. A numerical example illustrating the use of the obtained results for image analysis (edge detection) is presented in Section 2.5. Section 3 contains the proof of consistency against trend in location (Alternative 2).

\section{Consistency against change points (change surfaces) alternative}

\subsection{OnE-DimEnSIONAL CASE, $m=2$}

Let $\left\{x_{k}\right\}_{k=1}^{N}$ be a random sequence of size $N$. In this case the observation points are $k / N \in D:=(0,1], k=1, \ldots, N$. The problem is to test the null hypothesis

$$
H_{0}: \quad F_{1}(x)=F_{2}(x)=\cdots=F_{N}(x),
$$

where $F_{k}(x)$ is a continuous distribution function of the random variable $x_{k}, k=$ $1, \ldots, N$, against the alternative

$$
H_{2}: \quad F_{k}(x)=G_{1}(x), k / N \in D_{1}, \quad F_{k}(x)=G_{2}(x), k / N \in D_{2},
$$




$$
G_{1}(x) \geq G_{2}(x), x \in \mathbb{R}, \quad G_{1}\left(x_{0}\right)>G_{2}\left(x_{0}\right) \text { for some } x_{0},
$$

where $D_{1}$ and $D_{2}$ are (unknown) nonintersecting measurable sets such that $D_{1} \cup$ $D_{2}=(0,1]$. We assume that $G_{1}(x)$ and $G_{2}(x)$ are continuously differentiable, and the ties do not occur with probability 1 . Let $p^{(i)}$ be the number of interior points defined by:

$p^{(i)}:=p_{1}^{(i)}+p_{2}^{(i)}, \quad p_{j}^{(i)}:=\#\left\{k: k, k+1 \in K_{j}\right\}, K_{j}:=\left\{k: k / N \in D_{j}\right\}, j=1,2$.

All other points are called the boundary (change) points, there are $p^{(b)}:=N-p^{(i)}$ of them. Let us also assume that

$$
p^{(b)}<\text { const, } p_{1}^{(i)} / N \rightarrow \alpha, p_{2}^{(i)} / N \rightarrow 1-\alpha \quad \text { as } \quad N \rightarrow \infty, \quad 0<\alpha<1,
$$

where $\alpha=\lambda\left(D_{1}\right)$ is the Lebesgue measure of $D_{1}$. Let $R_{i}$ be the rank of the element $x_{i}, i=1, \ldots, N$. The test criterion is the statistic

$$
\nu_{N}:=\frac{1}{N-1} \sum_{k=1}^{N-1}\left(\frac{R_{k+1}-R_{k}}{N}\right)^{2}
$$

Let us calculate the moments of $\nu_{N}$ when the null hypothesis is true. We have

$E\left(\nu_{N}\right)=\frac{1}{N-1} \sum_{k=1}^{N-1} E\left(\frac{R_{k+1}-R_{k}}{N}\right)^{2}=\frac{1}{N(N-1)} \sum_{i=1}^{N} \sum_{\substack{j=1 \\ j \neq i}}^{N}\left(\frac{i-j}{N}\right)^{2}=\frac{1}{6}\left(1+\frac{1}{N}\right)$,

where we have used the fact that the distribution of the random variable $\left(R_{k+1}-\right.$ $\left.R_{k}\right)^{2}$ does not depend on $k$, and that all combinations of ranks in the pair $R_{k}, R_{k+1}$ : $R_{k}=i, R_{k+1}=j, i \neq j$, are equiprobable. The second moment of $\nu_{N}$ is given by:

$$
E\left(\nu_{N}^{2}\right)=\frac{(N+1)\left(5 N^{4}-17 N^{2}+18\right)}{180(N-1)^{2} N^{3}}=\frac{1}{36}(1+O(1 / N)) .
$$

¿From (2.1.5) and (2.1.6) it follows that

$$
\operatorname{var}\left(\nu_{N}\right)=1 /(36 N)+O\left(1 / N^{2}\right), \quad N \rightarrow \infty .
$$

Therefore, $\nu_{N}$ converges to $1 / 6$ in mean square as $N \rightarrow \infty$ if the null hypothesis holds. To investigate the behavior of $\nu_{N}$ under the alternative hypothesis, let $N \rightarrow \infty$. Since the portion of border points among all the points is of order 
$O(1 / N)$ (see $(2.1 .2 \mathrm{c}))$, we may write

$$
\begin{aligned}
E\left(\nu_{N}\right)= & \frac{1}{N-1} \sum_{k \in K_{1}} E\left(\frac{R_{k+1}-R_{k}}{N}\right)^{2}+\frac{1}{N-1} \sum_{k \in K_{2}} E\left(\frac{R_{k+1}-R_{k}}{N}\right)^{2} \\
= & \frac{\left|K_{1}\right|}{N-1} E\left(\frac{R_{l_{1}+1}-R_{l_{1}}}{N}\right)^{2}+\frac{\left|K_{2}\right|}{N-1} E\left(\frac{R_{l_{2}+1}-R_{l_{2}}}{N}\right)^{2}+O\left(\frac{1}{N}\right) \\
& \underset{N \rightarrow \infty}{\longrightarrow} \alpha \int_{0}^{1} \int_{0}^{1} f_{1}(x)(x-y)^{2} f_{1}(y) d x d y \\
& \quad+(1-\alpha) \int_{0}^{1} \int_{0}^{1} f_{2}(x)(x-y)^{2} f_{2}(y) d x d y \\
= & \frac{2}{3}-\frac{2 \alpha}{1-\alpha}\left\{\left(\int_{0}^{1} f_{1}(x) x d x\right)^{2}-\int_{0}^{1} f_{1}(x) x d x+\frac{1}{4 \alpha}\right\}:=E_{2} .
\end{aligned}
$$

Here $l_{1}$ and $l_{2}$ are arbitrary fixed indices such that $l_{1}, l_{1}+1 \in K_{1}, l_{2}, l_{2}+1 \in K_{2}$, and $\int_{a}^{b} f_{j}(r) d r, 0<a<b<1$, is the probability as $N \rightarrow \infty$ that the random variable $R_{i} / N, i \in K_{j}$, lies between $a$ and $b$. Existence of such functions $f_{j}(r)$ follows from the Glivenko theorem [R]. In (2.1.8), we have used the fact that $R_{k}$ and $R_{j}, k \neq j$, are asymptotically independent, and $\alpha f_{1}+(1-\alpha) f_{2}=1$. Similarly, let us show that $\operatorname{var}\left(\nu_{N}\right) \rightarrow 0$ if $H_{2}$ holds. We have

$$
\begin{aligned}
E\left(\nu_{N}^{2}\right) & =\frac{1}{(N-1)^{2}} E\left\{\left[\sum_{k \in K_{1}}\left(\frac{R_{k+1}-R_{k}}{N}\right)^{2}+\sum_{k \in K_{2}}\left(\frac{R_{k+1}-R_{k}}{N}\right)^{2}\right]^{2}\right\} \\
& =\sum_{p, q=1}^{2} \frac{1}{(N-1)^{2}} \sum_{k \in K_{p}} \sum_{j \in K_{q}} E_{k j}, \\
E_{k j}: & =E\left[\left(\frac{R_{k+1}-R_{k}}{N}\right)^{2}\left(\frac{R_{j+1}-R_{j}}{N}\right)^{2}\right] .
\end{aligned}
$$

Since

a) the number of terms for which $k=j$ or $|k-j|=1$ is proportional to $N$,

b) the portion of boundary points is of order $O(1 / N)$, and

c) $R_{k+1}-R_{k}$ and $R_{j+1}-R_{j},|k-j| \geq 2$, are asymptotically independent, we have

$$
\begin{aligned}
\frac{1}{(N-1)^{2}} \sum_{k \in K_{p}} \sum_{j \in K_{q}} E_{k j}=\frac{1}{(N-1)^{2}} \sum_{k \in K_{p}} \sum_{\substack{j \in K_{q} \\
|j-k| \geq 2}} E_{k j}+O\left(\frac{1}{N}\right) \\
=\left[\frac{\left|K_{p}\right|}{N-1} E\left(\frac{R_{m+1}-R_{m}}{N}\right)^{2}\right]\left[\frac{\left|K_{q}\right|}{N-1} E\left(\frac{R_{n+1}-R_{n}}{N}\right)^{2}\right]+O\left(\frac{1}{N}\right), \\
(2.1 .10)
\end{aligned}
$$

where $m$ and $n$ are arbitrary fixed indices such that $m, m+1 \in K_{p}, n, n+1 \in$ $K_{q},|m-n| \geq 2$. Formulas $(2.1 .8)-(2.1 .10)$ yield $E\left(\nu_{N}^{2}\right)=\left(E\left(\nu_{N}\right)\right)^{2}+O(1 / N)$, therefore $\operatorname{var}\left(\nu_{N}\right)=O(1 / N)$ as $N \rightarrow \infty$. Collecting (2.1.5), (2.1.7), and (2.1.8), we prove 
Theorem 2.1. One has $\nu_{N} \underset{N \rightarrow \infty}{\stackrel{\mathrm{ms}}{\longrightarrow}} 1 / 6$ if $H_{0}$ is true, and $\nu_{N} \underset{N \rightarrow \infty}{\stackrel{\mathrm{ms}}{\longrightarrow}} E_{2}$ if $H_{2}$ is true.

Remark 2.1. It is possible to relax the assumption $p^{(b)}<$ const as $N \rightarrow \infty$ and to require only $p^{(b)} / N=o(1)$ as $N \rightarrow \infty$. In this case, we have $\nu_{N} \rightarrow E_{2}$ with $\operatorname{var}\left(\nu_{N}\right)=o(1)$ as $N \rightarrow \infty$. For the proof of this fact note that the only difference in the argument is the replacement of the term $O(1 / N)$ in (2.1.8) and (2.1.10) by $o(1)$.

Theorem 2.2 below shows that the statistic $\nu_{N}$ can be used for testing $H_{0}$ against $\mathrm{H}_{2}$.

Theorem 2.2. Under assumption (2.1.2b) one has $E_{2}<1 / 6$.

Proof. We see from (2.1.8) that it is enough to prove inequality

$$
z^{2}-z+\frac{1}{4 \alpha}>\frac{1-\alpha}{4 \alpha}, \quad z:=\int_{0}^{1} f_{1}(r) r d r
$$

which is equivalent to $(z-1 / 2)^{2}>0$. To prove that $z \neq 1 / 2$, let us calculate the function $f_{1}(r)$. Denote $g_{1}(x):=G_{1}^{\prime}(x), g_{2}(x):=G_{2}^{\prime}(x)$. Pick an arbitrary index $i, 1 \leq i \leq N$, let $x$ be the value of the random variable at this point and let $r=R_{i} / N$ be the normalized rank of this value. By the Glivenko theorem, we have for large $N$

$$
r=\alpha G_{1}(x)+(1-\alpha) G_{2}(x):=G(x),
$$

or $x=G^{-1}(r)$. Here and below $G^{-1}(r)$ stands for the inverse of $G(x)$ where the inverse function is defined. It is defined, for example, for $r$ such that $r=$ $G(x), g(x):=G^{\prime}(x)>0$. Using (2.1.12) and taking into account that $1 / g\left(G^{-1}(r)\right)$ is the Jacobian of the transformation $x \rightarrow r, x=G^{-1}(r)$, we get for $r$ such that $r=G(x)$ and $g(x)>0$ :

$$
f_{1}(r)=\frac{g_{1}\left(G^{-1}(r)\right)}{g\left(G^{-1}(r)\right)} .
$$

Let $E^{\prime}$ be the set $\{r: 0 \leq r \leq 1, G(x)=r, g(x)=0\}$. By Sard's theorem [S], $\lambda\left(E^{\prime}\right)=0$, where $\lambda(E)$ is the usual Lebesgue measure in $\mathbb{R}^{1}$. Let $E$ be the complement of $E^{\prime}$ in $[0,1], \lambda(E)=1$. Fix any $\epsilon>0$ and denote by $E_{\epsilon}^{\prime}$ any open covering of $E^{\prime}$, such that $\lambda\left(E_{\epsilon}^{\prime}\right)=\epsilon$. Let $E_{\epsilon}:=[0,1] \backslash E_{\epsilon}^{\prime}$ and $\mathbb{R}_{\epsilon}:=G^{-1}\left(E_{\epsilon}\right)$. Consider the integral

$$
\int_{E_{\epsilon}} f_{1}(r) r d r=\int_{E_{\epsilon}} \frac{g_{1}\left(G^{-1}(r)\right)}{g\left(G^{-1}(r)\right)} r d r=\int_{\mathbb{R}_{\epsilon}} \frac{g_{1}(x)}{g(x)} G(x) g(x) d x=\int_{\mathbb{R}_{\epsilon}} G(x) g_{1}(x) d x .
$$

Let $\epsilon \rightarrow 0$ in the above formula. Then the limit of the left side is denoted by $\int_{0}^{1} f(r) r d r$ (since $\lambda(E)=1$ ). This limit does exist since the limit of the right side exists and is equal to $\int_{G^{-1}(E)} G(x) g_{1}(x) d x=\int G(x) g_{1}(x) d x$, where $\int:=\int_{\mathbb{R}}$. The last equation holds because $g_{1}(x)=0$ on $G^{-1}\left(E^{\prime}\right)$ and $\mathbb{R}=G^{-1}(E) \cup G^{-1}\left(E^{\prime}\right)$. Therefore we conclude that

$$
z:=\int_{0}^{1} f_{1}(r) r d r=\int G(x) g_{1}(x) d x=\frac{\alpha}{2}+(1-\alpha) \int G_{2} g_{1} d x .
$$


Using that $G_{1}(x) \geq G_{2}(x), G_{1}\left(x_{0}\right)>G_{2}\left(x_{0}\right)$ and continuity of $g_{1}(x)$, we have $\int G_{2} g_{1} d x<\int G_{1} g_{1} d x=1 / 2$. This together with (2.1.14) yields $z<1 / 2$. Theorem 2.2 is proved.

Remark 2.2. From the proof of Theorem 2.2 it follows that $E_{2}=1 / 6$ if and only if $G_{1}(x) \equiv G_{2}(x)$, i.e. when $H_{0}=H_{2}$. Here $E_{2}$ is defined in (2.1.8).

Remark 2.3. From the argument below (2.1.14) we see that to prove the inequality $z<1 / 2$ it is sufficient to have only $\int G_{2} g_{1} d x<1 / 2$. Thus Theorem 2.2 holds under condition weaker than (2.1.2b), which can be replaced by $P\left\{\xi_{1} \leq \xi_{2}\right\}>1 / 2$, where $\xi_{k}$ is the random variable with the distribution function $G_{k}, k=1,2$.

Using Theorems 2.1 and 2.2, let us construct the following test of randomness. Fix the probability $\epsilon, 0<\epsilon<1$, of the type I error and let the rejection region be $\left\{x: x<A_{0}\right\}$, where the threshold $A_{0}$ is determined from the equation $P\left\{\nu_{N}<\right.$ $\left.A_{0} \mid H_{0}\right\}=\epsilon$. Consistency of the proposed test easily follows. Indeed, since $E_{2}<1 / 6$ (Theorem 2.2) and $A_{0} \rightarrow 1 / 6$ as $N \rightarrow \infty$ (according to the choice of $A_{0}$ and Theorem 2.1), we have using the Chebyshev inequality and assuming that $H_{2}$ holds

$$
P\left\{\nu_{N} \geq A_{0}\right\} \leq P\left\{\left|\nu_{N}-E_{2}\right| \geq A_{0}-E_{2}\right\} \leq \frac{\operatorname{var}\left(\nu_{N}\right)}{\left(A_{0}-E_{2}\right)^{2}}=O\left(\frac{1}{N}\right) \text { as } N \rightarrow \infty .
$$

If $N$ is sufficiently large, the threshold $A_{0}$ is found using the asymptotic normality of $\nu_{N}$ with the mean value $1 / 6$ and variance $1 /(36 N)$. If $N$ is small, a convenient way to compute $A_{0}$ is by using the Monte-Carlo method [KW].

\subsection{One-Dimensional Case, $m>2$}

In this section we prove that the test based on $\nu_{N}$ is consistent against an alternative more general than $H_{2}$. Let us fix $m \geq 2$ and define the alternative hypothesis $H_{m}$.

$$
H_{m}: \quad F_{k}(x)=G_{l}(x), k / N \in D_{l}, l=1, \ldots, m,
$$

where we assume that

$$
\begin{gathered}
G_{l}(x) \geq G_{l+1}(x), x \in \mathbb{R} ; \quad \exists x_{0 l}: G_{l}\left(x_{0 l}\right)>G_{l+1}\left(x_{0 l}\right), l=1, \ldots, m-1, \\
\bigcup_{l=1}^{m} D_{l}=D=(0,1], D_{i} \cap D_{j}=\varnothing, i \neq j,
\end{gathered}
$$

$G_{l}(x)$ are continuously differentiable and $D_{l}$ are measurable, $l=1, \ldots, m$. Similarly to (2.1.3), let us introduce the interior and boundary points

$$
\begin{gathered}
p_{l}^{(i)}:=\#\left\{k: k, k+1 \in K_{l}\right\}, K_{l}:=\left\{k: k / N \in D_{l}\right\}, l=1, \ldots, m ; \\
p^{(b)}:=N-\sum_{l=1}^{m} p_{l}^{(i)},
\end{gathered}
$$

and assume that

$$
\begin{gathered}
p_{l}^{(i)} / N \rightarrow \alpha_{l}=\lambda\left(D_{l}\right) \text { as } N \rightarrow \infty, 0<\alpha_{l}<1, l=1, \ldots, m ; \\
\sum_{l=1}^{m} \alpha_{l}=1 ; \quad p^{(b)}<\text { const. }
\end{gathered}
$$


Similarly to (2.1.8), (2.1.12), and (2.1.13), we have

$$
\begin{gathered}
E\left(\nu_{N}\right) \underset{N \rightarrow \infty}{\longrightarrow} \sum_{l=1}^{m} \alpha_{l} \int_{0}^{1} \int_{0}^{1} f_{l}(x)(x-y)^{2} f_{l}(y) d x d y:=E_{m}\left(\alpha_{1}, \ldots, \alpha_{m} ; G_{1}, \ldots, G_{m}\right), \\
f_{l}(r):=\frac{g_{l}\left(G^{-1}(r)\right)}{g\left(G^{-1}(r)\right)} \quad \text { for almost all } r \in[0,1], \\
G(x):=\alpha_{1} G_{1}(x)+\cdots+\alpha_{m} G_{m}(x), \quad g(x):=G^{\prime}(x) .
\end{gathered}
$$

Theorem 2.3. Under assumptions (2.2.1b, c) and (2.2.3) one has

$$
E_{m}\left(\alpha_{1}, \ldots, \alpha_{m} ; G_{1}, \ldots, G_{m}\right)<E_{m-1}\left(\alpha_{1}, \ldots, \alpha_{m-2}, \alpha_{m-1}+\alpha_{m} ; G_{1}, \ldots, G_{m-1}\right) \text {. }
$$

Proof. Substitution of (2.2.5) into (2.2.4) and the change of variables $x=G^{-1}(r)$ yields

$$
E_{m}\left(\alpha_{1}, \ldots, \alpha_{m} ; G_{1}, \ldots, G_{m}\right)=2\left\{\frac{1}{3}-\sum_{l=1}^{m} \alpha_{l}\left(\int G(x) g_{l}(x) d x\right)^{2}\right\} .
$$

Defining

$$
\tilde{G}(x):=\alpha_{1} G_{1}(x)+\cdots+\alpha_{m-2} G_{m-2}(x)+\left(\alpha_{m-1}+\alpha_{m}\right) G_{m-1}(x), \tilde{g}(x):=\tilde{G}^{\prime}(x),
$$

we obtain that (2.2.6) is equivalent to

$$
\begin{aligned}
\sum_{l=1}^{m} \alpha_{l}\left(\int G(x) g_{l}(x) d x\right)^{2} & >\sum_{l=1}^{m-2} \alpha_{l}\left(\int \tilde{G}(x) g_{l}(x) d x\right)^{2} \\
& +\left(\alpha_{m-1}+\alpha_{m}\right)\left(\int \tilde{G}(x) g_{m-1}(x) d x\right)^{2} .
\end{aligned}
$$

Define $H(x):=G_{m}(x)-G_{m-1}(x), h(x):=H^{\prime}(x)$. Since $G(x)=\tilde{G}(x)+\alpha_{m} H(x)$ and $g_{m}(x)=g_{m-1}(x)+h(x)$, we have from (2.2.7) and (2.2.8)

$$
\begin{aligned}
& 2 \sum_{l=1}^{m-1} \alpha_{l} \int \tilde{G} g_{l} d x \int \alpha_{m} H g_{l} d x+\sum_{l=1}^{m-1} \alpha_{l}\left(\int \alpha_{m} H g_{l} d x\right)^{2} \\
& \quad+\alpha_{m}\left(\int\left(\tilde{G}+\alpha_{m} H\right)\left(g_{m-1}+h\right) d x\right)^{2}>\alpha_{m}\left(\int \tilde{G} g_{m-1} d x\right)^{2} .
\end{aligned}
$$

We have $H(x)=G_{m}(x)-G_{m-1}(x) \rightarrow 1-1=0$ as $x \rightarrow \infty$ and $H(x) \rightarrow 0-0=0$ as $x \rightarrow-\infty$, therefore $\int H h d x=\int H d H=\left(H^{2}(+\infty)-H^{2}(-\infty)\right) / 2=0$. Hence

$$
\begin{aligned}
\left(\int\left(\tilde{G}+\alpha_{m} H\right)\left(g_{m-1}+h\right) d x\right)^{2} & \left(\int \tilde{G} g_{m-1} d x+\int \alpha_{m} H g_{m-1} d x+\int \tilde{G} h d x\right)^{2} \\
= & \left(\int \tilde{G} g_{m-1} d x\right)^{2}+\left(\int \alpha_{m} H g_{m-1} d x+\int \tilde{G} h d x\right)^{2} \\
& +2 \int \tilde{G} g_{m-1} d x\left(\int \alpha_{m} H g_{m-1} d x+\int \tilde{G} h d x\right) .
\end{aligned}
$$


Substitution into (2.2.9) gives

$$
\begin{aligned}
2 \sum_{l=1}^{m-1} \alpha_{l} \int \tilde{G} g_{l} d x \int \alpha_{m} H g_{l} d x+2 \alpha_{m} \int \tilde{G} g_{m-1} d x\left(\int \alpha_{m} H g_{m-1} d x\right. & \\
& \left.+\int \tilde{G} h d x\right)+A>0
\end{aligned}
$$

where

$$
A:=\sum_{l=1}^{m-1} \alpha_{l}\left(\int \alpha_{m} H g_{l} d x\right)^{2}+\alpha_{m}\left(\int \alpha_{m} H g_{m-1} d x+\int \tilde{G} h d x\right)^{2} \geq 0 .
$$

We have

$$
\begin{aligned}
\int \alpha_{m} H g_{m-1} d x+\int \tilde{G} h d x & =\int \alpha_{m} H g_{m-1} d x-\int H \tilde{g} d x=\int H\left(\alpha_{m} g_{m-1}-\tilde{g}\right) d x \\
& =-\int H\left(\alpha_{1} g_{1}+\cdots+\alpha_{m-1} g_{m-1}\right) d x
\end{aligned}
$$

The last equation and (2.2.10) imply that it is sufficient to prove

$$
\sum_{l=1}^{m-1} \alpha_{l} \int \tilde{G} g_{l} d x \int H g_{l} d x \geq \int \tilde{G} g_{m-1} d x \int H\left(\alpha_{1} g_{1}+\cdots+\alpha_{m-1} g_{m-1}\right) d x,
$$

where we cancelled $2 \alpha_{m}>0$. This inequality is equivalent to the following one

$$
\sum_{l=1}^{m-1} \alpha_{l}\left\{\int \tilde{G}\left(g_{l}-g_{m-1}\right) d x\right\} \int H g_{l} d x \geq 0 .
$$

Integrating by parts the expression in braces, we get

$$
\begin{aligned}
\int \tilde{G}\left(g_{l}-g_{m-1}\right) d x & =\left.\tilde{G}\left(G_{l}-G_{m-1}\right)\right|_{-\infty} ^{\infty}-\int\left(G_{l}-G_{m-1}\right) \tilde{g} d x \\
& =\int\left(G_{m-1}-G_{l}\right) \tilde{g} d x \leq 0,1 \leq l \leq m-1 .
\end{aligned}
$$

The last inequality holds because $\tilde{g}=\alpha_{1} g_{1}+\cdots+\left(\alpha_{m-1}+\alpha_{m}\right) g_{m-1} \geq 0$ and $G_{m-1} \leq G_{l}, 1 \leq l \leq m-1$. Together with the inequality $H=G_{m}-G_{m-1} \leq 0$, this proves (2.2.12). ¿From (2.2.11) and (2.2.12) we see that we proved (2.2.10) with " $\geq$ " in place of " $>$ ". To prove the strict inequality, it is sufficient to prove that $\int \bar{H} g_{m-1} d x<0$, which implies $A>0$. We have, using (2.2.1b)

$$
\int H g_{m-1} d x=\int\left(G_{m}-G_{m-1}\right) d G_{m-1}<0
$$

Theorem 2.3 is proved.

Applying inequality (2.2.6) repeatedly and using Theorem 2.2, we obtain

$$
\begin{aligned}
E\left(\nu_{N}\right) & \underset{N \rightarrow \infty}{\longrightarrow} E_{m}\left(\alpha_{1}, \ldots, \alpha_{m} ; G_{1}, \ldots, G_{m}\right) \\
& <E_{2}\left(\alpha_{1}, \alpha_{2}+\cdots+\alpha_{m} ; G_{1}, G_{2}\right) \\
& =E_{2}\left(\alpha_{1}, 1-\alpha_{1} ; G_{1}, G_{2}\right)=E_{2}<1 / 6 .
\end{aligned}
$$

As in the previous section, it is easy to prove that $\operatorname{var}\left(\nu_{N}\right)=O(1 / N)$ if $H_{m}$ holds. Thus the test of randomness based on $\nu_{N}$ is also consistent against $H_{m}$ for any fixed $m \geq 2$ with probability of type II error being of order $O(1 / N), N \rightarrow \infty$. 


\subsection{Multidimensional CASE, FiXed DESign MOdEL}

Let the given data be $x_{k_{1}, \ldots, k_{d}}, 1 \leq k_{i} \leq \beta_{i} N, i=1, \ldots, d$, where $\beta_{i}$ are fixed integers. In this case the observation points are

$$
\frac{1}{N}\left(k_{1}, \ldots, k_{d}\right) \in D:=\left(0, \beta_{1}\right] \times \cdots \times\left(0, \beta_{d}\right] \subset \mathbb{R}^{d} .
$$

Let us denote $k:=\left(k_{1}, \ldots, k_{d}\right)$ and $B_{N}:=\left\{k \in \mathbb{N}^{d}: 1 \leq k_{i} \leq \beta_{i} N, i=1, \ldots, d\right\}$. The problem is to test the null hypothesis

$$
H_{0}: \quad F_{k}(x)=F_{j}(x) \quad \text { for every } k, j \in B_{N},
$$

where $F_{k}(x)$ is a continuous distribution function of the random variable $x_{k}, k \in$ $B_{N}$, against the alternative

$$
\begin{aligned}
& H_{m}: F_{k}(x)=G_{j}(x), k / N \in D_{j}, j=1, \ldots, m, \\
& \bigcup_{j=1}^{m} D_{j}=D, D_{i} \cap D_{j}=\varnothing, i \neq j .
\end{aligned}
$$

Here the functions $G_{j}(x)$ satisfy the same conditions as in Section 2.2 (see $(2.2 .1 \mathrm{~b}$ ) and below) and $D_{j}$ are measurable, $j=1, \ldots, m$. For an arbitrary multiindex $k \in B_{N}$ we define the set of multiindices neighboring to $k$ by the formula $L(k):=$ $\left\{l \in B_{N}: l \neq k, \max _{1 \leq i \leq d}\left|l_{i}-k_{i}\right|=1\right\}$. We see that if $k$ is strictly inside $B_{N}$, the number of elements in $L(k)$ is independent of $k$ and is equal to $3^{d}-1$. Similarly to (2.1.3) and (2.2.2), we introduce the interior and boundary points

$$
\begin{gathered}
p_{j}^{(i)}:=\#\left\{k \in \mathbb{N}^{d}: k, L(k) \in K_{j}\right\}, K_{j}:=\left\{k \in \mathbb{N}^{d}: k / N \in D_{j}\right\}, j=1, \ldots, m ; \\
p^{(b)}:=N^{d}-\sum_{j=1}^{m} p_{j}^{(i)}
\end{gathered}
$$

and assume that

$$
\begin{gathered}
p_{j}^{(i)} / \hat{N} \rightarrow \alpha_{j}:=\lambda\left(D_{j}\right) \text { as } N \rightarrow \infty, 0<\alpha_{j}<1, j=1, \ldots, m ; \\
\sum_{j=1}^{m} \alpha_{j}=1 ; \quad p^{(b)}<\operatorname{const} N^{d-1} .
\end{gathered}
$$

Here $\lambda(\cdot)$ is the Lebesgue measure in $\mathbb{R}^{d}$ and $\hat{N}:=\beta_{1} \cdot \ldots \cdot \beta_{d} N^{d}$ is the number of lattice points. Let $R_{i}$ be the rank of the element $x_{i}, i \in B_{N}$. The test criterion is the statistic

$$
\nu_{N}:=\frac{1}{M_{N}} \sum_{k \in B_{N}} \sum_{l \in L(k)}\left(\frac{R_{k}-R_{l}}{\hat{N}}\right)^{2},
$$

where $M_{N}$ is the number of elements in double sum (2.3.3). We see that $M_{N}=$ $\left(3^{d}-1\right) \hat{N}(1+O(1 / N))$ as $N \rightarrow \infty$. Note also that each pair $\left(R_{k}, R_{l}\right), k \neq l$, regardless of order, appears in the double sum in (2.3.3) twice. Similarly to (2.1.5) 
and (2.1.6), the first two moments of $\nu_{N}$ when the null hypothesis is true are given by

$$
E\left(\nu_{N}\right)=1 / 6+1 /(6 \hat{N}), \quad E\left(\nu_{N}^{2}\right)=1 / 36+O\left(N^{-d}\right) .
$$

This yields

$$
\operatorname{var}\left(\nu_{N}\right)=E\left(\nu_{N}^{2}\right)-\left(E\left(\nu_{N}\right)\right)^{2}=O\left(N^{-d}\right), N \rightarrow \infty .
$$

Now let us consider the behavior of $\nu_{N}$ under the alternative hypothesis $H_{m}$. Since the portion of border points among all the points is of order $O(1 / N)$, we may write similarly to (2.1.8) and (2.2.4)

$$
\begin{aligned}
& E\left(\nu_{N}\right)=\sum_{j=1}^{m} \frac{1}{M_{N}} \sum_{k \in K_{j}} \sum_{l \in L(k)} E\left(\frac{R_{k}-R_{l}}{\hat{N}}\right)^{2}=\sum_{j=1}^{m} \frac{\left|K_{j}\right|}{\hat{N}} E\left(\frac{R_{k_{j}}-R_{l_{j}}}{\hat{N}}\right)^{2}+O\left(\frac{1}{N}\right) \\
& \underset{N \rightarrow \infty}{\longrightarrow} \sum_{j=1}^{m} \alpha_{j} \int_{0}^{1} \int_{0}^{1} f_{j}(x)(x-y)^{2} f_{j}(y) d x d y=E_{m}\left(\alpha_{1}, \ldots, \alpha_{m} ; G_{1}, \ldots, G_{m}\right)=E_{m} .
\end{aligned}
$$

where $k_{j}$ and $l_{j}$ are arbitrary different multiindices such that $k_{j}, l_{j} \in K_{j}, j=$ $1, \ldots m$. As in Section 2.1, it is easy to show that $\operatorname{var}\left(\nu_{N}\right)=O(1 / N)$ as $N \rightarrow \infty$. Collecting (2.3.4) - (2.3.6), we prove

Theorem 2.4. One has $\nu_{N} \underset{N \rightarrow \infty}{\stackrel{\mathrm{ms}}{\longrightarrow}} 1 / 6$ if $H_{0}$ is true, and $\nu_{N} \underset{N \rightarrow \infty}{\stackrel{\mathrm{ms}}{\longrightarrow}} E_{m}$ if $H_{m}$ is true.

Using Theorem 2.2, we see that the statistic $\nu_{N}$ defined by (2.3.3) can be used for testing $H_{0}$ against $H_{m}$ in the multidimensional case. Consistency of the test follows easily from Theorems 2.2 and 2.4. Note that the probability of a type II error is $O(1 / N)$ as $N \rightarrow \infty$. The threshold $A_{0}$ is determined from the equation $P\left\{\nu_{N}<A_{0} \mid H_{0}\right\}=\epsilon$. If $N$ is sufficiently large, $A_{0}$ is found using the asymptotic normality of $\nu_{N}$ [CO]. If $N$ is small, a convenient way to compute $A_{0}$ is by using the Monte-Carlo method [KW].

\subsection{RANDOM DESIGN MODEL}

Let the observation points $\left\{\rho_{k}\right\}_{k=1}^{N}$ be randomly chosen inside an open bounded domain $D \subset \mathbb{R}^{d}, d \geq 1$, and let $\left\{x_{k}\right\}_{k=1}^{N}$ be a set of corresponding observations. The observation points are called random if they are independent and identically distributed inside $D$ with constant probability density. The problem is to test the null hypothesis

$$
H_{0}: \quad F_{1}(x)=F_{2}(x)=\cdots=F_{N}(x),
$$

where $F_{k}(x)$ is a continuous distribution function of the random variable observed at the point $\rho_{k}, k=1, \ldots, N$, against the alternative

$$
\begin{gathered}
H_{m}: \quad F_{k}(x)=G_{i}(x) \text { for } \rho_{k} \in D_{i}, \\
\lambda\left(D_{j}\right)>0, j=1, \ldots, m, \bigcup_{j=1}^{m} D_{j}=D, D_{i} \cap D_{j}=\varnothing, i \neq j,
\end{gathered}
$$


where we assume, as usual, that the partition $D_{j}, j=1, \ldots, m$, and $m \geq 2$ are fixed. Here $\lambda(\cdot)$ is the Lebesgue measure in $\mathbb{R}^{d}$, and the functions $G_{i}$ satisfy the same conditions as in Section 2.2. The test criterion is the statistic

$$
\nu_{N}:=\frac{1}{N} \sum_{k=1}^{N}\left(\frac{R_{n(k)}-R_{k}}{N}\right)^{2},
$$

where $n(k)$ is the index of the point closest to $\rho_{k},\left|\rho_{n(k)}-\rho_{k}\right|=\min _{\substack{1 \leq j \leq N \\ j \neq k}}\left|\rho_{j}-\rho_{k}\right|$. Note that $n(k)$ is unique with probability 1 . The first two moments of $\nu_{N}$ can be easily computed under the assumption that $H_{0}$ holds:

$$
E\left(\nu_{N}\right)=1 / 6+1 /(6 N), \quad E\left(\nu_{N}^{2}\right)=1 / 36+O(1 / N) .
$$

In (2.4.4), we used that

a) for a fixed index $k$, there exists a number $\gamma, 0<\gamma<\infty$, which is independent of $k,\left\{\rho_{k}\right\}_{k=1}^{N}$, and $N$, such that the number of indices $l$ for which $\{k, n(k)\} \cap\{l, n(l)\} \neq \varnothing$ is bounded by $\gamma$, and

b) the random variables $R_{n(k)}-R_{k}$ and $R_{n(l)}-R_{l}$ for $k$ and $l$ such that $\{k, n(k)\} \cap\{l, n(l)\}=\varnothing$ are asymptotically independent.

Equations (2.4.4) imply

$$
\operatorname{var}\left(\nu_{N}\right)=O(1 / N), N \rightarrow \infty .
$$

Now let us study the asymptotic behavior of $\nu_{N}$ under the assumption that $H_{m}$ holds. Define $\Gamma:=\bigcup_{i=1}^{m} \partial D_{i}$, where $\partial D_{i}$ is the boundary of $D_{i}$. Fix any $\epsilon>0$ and define $V_{\epsilon}:=\{s \in D: \operatorname{dist}(s, \Gamma) \leq \epsilon\}$. We assume that $\Gamma$ is sufficiently smooth, so that $\lambda\left(V_{\epsilon}\right) \rightarrow 0$ as $\epsilon \rightarrow 0$. We have $P\left\{\lim _{N \rightarrow \infty}\left(\max _{1 \leq k \leq N} \min _{\substack{1 \leq j \leq N \\ j \neq k}}\left|\rho_{k}-\rho_{j}\right|\right)=\right.$ $0\}=1$. Using this, fix any $\delta>0$ and find $N_{1}$ such that

$$
P\left\{\max _{1 \leq k \leq N} \min _{\substack{1 \leq j \leq N \\ j \neq k}}\left|\rho_{k}-\rho_{j}\right|>\epsilon\right\}<\delta, \quad N \geq N_{1} .
$$

Let us introduce some notation

$$
\begin{gathered}
\rho:=\left\{\rho_{k}\right\}_{k=1}^{N}, \quad V_{i}:=D_{i} \cap V_{\epsilon}, \quad U_{i}:=D_{i} \backslash V_{i}, \\
d_{i}:=\lambda\left(D_{i}\right) / \lambda(D), \quad v_{\epsilon}:=\lambda\left(V_{\epsilon}\right) / \lambda(D), \quad v_{i}:=\lambda\left(V_{i}\right) / \lambda(D), \quad u_{i}:=\lambda\left(U_{i}\right) / \lambda(D), \\
\tilde{U}_{i}:=\tilde{U}_{i}(\rho):=\#\left\{k: \rho_{k} \in U_{i}\right\}, \quad \tilde{V}_{i}:=\tilde{V}_{i}(\rho):=\#\left\{k: \rho_{k} \in V_{i}\right\}, \\
\tilde{D}_{i}:=\tilde{D}_{i}(\rho):=\#\left\{k: \rho_{k} \in D_{i}\right\}, \quad \Delta_{N}:=\Delta_{N}(\rho):=\max _{\substack { 1 \leq k \leq N \\
\begin{subarray}{c}{1 \leq j \leq N \\
j \neq k{ 1 \leq k \leq N \\
\begin{subarray} { c } { 1 \leq j \leq N \\
j \neq k } }\end{subarray}}\left|\rho_{k}-\rho_{j}\right|, \\
\mathcal{P}(\delta, N):=\left\{\rho:\left|\frac{\tilde{V}_{i}}{N}-v_{i}\right|<\delta,\left|\frac{\tilde{U}_{i}}{N}-u_{i}\right|<\delta, 1 \leq i \leq m\right\} .
\end{gathered}
$$

Recall that we assume $N \gg m$. Since the distribution of points inside $D$ is assumed to be random, one can easily get $P\{\rho \notin \mathcal{P}(\delta, N)\} \rightarrow 0$ as $N \rightarrow \infty$. Thus,

$$
\exists N_{2} \text { such that } P\{\rho \notin \mathcal{P}(\delta, N)\}<\delta, N \geq N_{2} .
$$


Using properties of conditional expectation, we get

$$
\begin{gathered}
E\left(\nu_{N}\right)=E\left(\nu_{N} \mid \rho \in \mathcal{P}(\delta, N), \Delta_{N} \leq \epsilon\right) P\left\{\rho \in \mathcal{P}(\delta, N), \Delta_{N} \leq \epsilon\right\}+ \\
E\left(\nu_{N} \mid \rho \notin \mathcal{P}(\delta, N), \Delta_{N} \leq \epsilon\right) P\left\{\rho \notin \mathcal{P}(\delta, N), \Delta_{N} \leq \epsilon\right\}+E\left(\nu_{N} \mid \Delta_{N}>\epsilon\right) P\left\{\Delta_{N}>\epsilon\right\}
\end{gathered}
$$

The last equation together with (2.4.6) and (2.4.8) implies

$$
\left|E\left(\nu_{N}\right)-E\left(\nu_{N} \mid W\right) P\{W\}\right|<2 \delta, N \geq N_{0}:=\max \left(N_{1}, N_{2}\right)
$$

where the event $\{W\}$ is defined as $\{W\}:=\left\{\rho \in \mathcal{P}(\delta, N), \Delta_{N} \leq \epsilon\right\}$. Pick an arbitrary $\rho$ such that $\rho \in \mathcal{P}(\delta, N)$ and $\Delta_{N} \leq \epsilon$. Using (2.4.3), we get

$$
\begin{aligned}
E\left(\nu_{N} \mid \rho\right)= & \sum_{i=1}^{m} \frac{1}{N} \sum_{k: \rho_{k} \in U_{i}} E\left(\left(\frac{R_{n(k)}-R_{k}}{N}\right)^{2} \mid \rho\right)+ \\
& \sum_{i=1}^{m} \frac{1}{N} \sum_{k: \rho_{k} \in V_{i}} E\left(\left(\frac{R_{n(k)}-R_{k}}{N}\right)^{2} \mid \rho\right) .
\end{aligned}
$$

Since the number of observations inside $V_{\epsilon}$ is bounded by $\left(v_{\epsilon}+m \delta\right) N$, and the distribution of the random variable $\left(\frac{R_{n(k)}-R_{k}}{N}\right)^{2}$ does not depend on $k$, provided that $\rho_{k} \in U_{i}$ (according to the choice of $\rho$ and $U_{i}$, if $\rho_{k} \in U_{i} \subset D_{i}$, then $\rho_{n(k)} \in D_{i}$ ), we obtain from (2.4.7) and (2.4.10):

$$
\left|E\left(\nu_{N} \mid \rho\right)-\sum_{i=1}^{m} \frac{\tilde{U}_{i}}{N} E\left(\left(\frac{R_{n\left(k_{i}\right)}-R_{k_{i}}}{N}\right)^{2} \mid \rho\right)\right| \leq v_{\epsilon}+m \delta .
$$

Let $\rho_{0}$ be the distribution of observation points such that $\tilde{V}_{i}\left(\rho_{0}\right) / N=v_{i}, \tilde{U}_{i}\left(\rho_{0}\right) / N$ $=u_{i}, i=1, \ldots, m$. We will use two facts.

a) The distribution function of the random variable $\left(\frac{R_{n\left(k_{i}\right)}-R_{k_{i}}}{N}\right)^{2}, \rho_{k_{i}} \in U_{i}$, does not depend on the location of observation points $\rho_{k}$ inside the sets $U_{i}$ and $V_{i}, i=1, \ldots, m$, it depends only on parameters $\tilde{U}_{i}(\rho)+\tilde{V}_{i}(\rho), i=$ $1, \ldots, m$.

b) Consider the set of observations $\left\{x_{k}\right\}_{k=1}^{N}$ corresponding to $\rho$ and change arbitrarily the values of observations at no more that $\delta N$ points in each of the sets $U_{i}$ and $V_{i}, i=1, \ldots, m$. We obtain a new sequence $\left\{\hat{x}_{k}\right\}_{k=1}^{N}$ and a new set of corresponding ranks $\left\{\hat{R}_{k}\right\}_{k=1}^{N}$. If the observation has not been changed at the point $\rho_{k}$, then $\left|\left(R_{k}-\hat{R}_{k}\right) / N\right| \leq 4 m \delta$.

According to the choice of $\rho$ and $\rho_{0}$, we get using a), b), and the triangle inequality

$$
\left|E\left(\left(\frac{R_{n\left(k_{i}\right)}-R_{k_{i}}}{N}\right)^{2} \mid \rho\right)-E\left(\left(\frac{R_{n\left(k_{i}\right)}-R_{k_{i}}}{N}\right)^{2} \mid \rho_{0}\right)\right| \leq 32 m \delta .
$$

Inequalities (2.4.7), (2.4.11), and (2.4.12) imply

$$
\left|E\left(\nu_{N} \mid \rho\right)-\sum_{i=1}^{m} d_{i} E\left(\left(\frac{R_{n\left(k_{i}\right)}-R_{k_{i}}}{N}\right)^{2} \mid \rho_{0}\right)\right| \leq 2 v_{\epsilon}+34 m \delta .
$$


Since the distribution of observations $\rho_{0}$ is fixed, we obtain similarly to (2.1.8) and $(2.2 .4)$

$$
\lim _{N \rightarrow \infty} \sum_{i=1}^{m} d_{i} E\left(\left(\frac{R_{n\left(k_{i}\right)}-R_{k_{i}}}{N}\right)^{2} \mid \rho_{0}\right)=E_{m}\left(d_{1}, \ldots, d_{m} ; G_{1}, \ldots, G_{m}\right):=E_{m} .
$$

Hence

$$
\left|E\left(\nu_{N} \mid \rho\right)-E_{m}\right| \leq 2 v_{\epsilon}+34 m \delta+o(1), N \rightarrow \infty .
$$

Note that $o(1), N \rightarrow \infty$, in (2.4.14) is independent of $\rho$. Recalling the definition of the event $W$ (see below (2.4.9)), we get $\left|E\left(\nu_{N} \mid W\right)-E_{m}\right| \leq 2 v_{\epsilon}+34 m \delta+o(1)$. This together with (2.4.9) and an obvious inequality $P\{W\} \geq 1-P\{\rho \notin \mathcal{P}(\delta, N)\}-$ $P\left\{\Delta_{N}>\epsilon\right\} \geq 1-2 \delta$ implies

$$
\left|E\left(\nu_{N}\right)-E_{m}\right| \leq 4 \delta+\left(2 v_{\epsilon}+34 m \delta+o(1)\right)(1+2 \delta), N \rightarrow \infty .
$$

Taking the limit as $N \rightarrow \infty$ and using that $\epsilon, \delta>0$ were arbitrary, we conclude

$$
\lim _{N \rightarrow \infty} E\left(\nu_{N}\right)=E_{m}
$$

Similarly, one can show that $\operatorname{var}\left(\nu_{N}\right) \rightarrow 0$ as $N \rightarrow \infty$. Together with (2.4.4) and (2.4.5), this yields

Theorem 2.5. One has $\nu_{N} \underset{N \rightarrow \infty}{\stackrel{\mathrm{ms}}{\longrightarrow}} 1 / 6$ if $H_{0}$ is true, and $\nu_{N} \underset{N \rightarrow \infty}{\stackrel{\mathrm{ms}}{\longrightarrow}} E_{m}$ if $H_{m}$ is true.

Using Theorem 2.2, we see that the statistic $\nu_{N}$ defined by (2.4.3) can be used for testing $H_{0}$ against $H_{m}$ in the case of random observation points. Consistency of the test follows easily from Theorems 2.2 and 2.5 .

\subsection{NUMERICAL EXPERIMENTS}

The results obtained in previous sections can be used in many applications: in particular, in image processing for edge detection. Let us describe the algorithm for edge detection based on these results. In image processing, the data are intensities of grey level specified at each pixel, i.e. at the nodes of two-dimensional square grid (image). An edge (discontinuity of a signal) can be defined as follows: the grey level is relatively consistent in each of the two adjacent extensive regions, and changes abruptly as the border between two regions is crossed $[\mathrm{P}, \mathrm{RK}]$. Consider $N \times N$ window $B_{N}$ sliding over the image. For each position of the window we want to make a decision: whether $\Gamma \cap B_{N}=\varnothing$ or not, where $\Gamma$ is an edge. If $\Gamma \cap B_{N} \neq \varnothing$, then $\Gamma$ divides $B_{N}$ into two sets $K_{1}$ and $K_{2}$, such that the values of grey level in one set are stochastically larger than in the other set, hence the hypothesis $H_{2}$ (or, more generally, $H_{m}$ ) takes place. If $\Gamma \cap B_{N}=\varnothing$, then the grey level is approximately constant inside $B_{N}$ and the hypothesis $H_{0}$ takes place. Thus, the choice between " $\Gamma \cap B_{N}=\varnothing$ " $\left(H_{0}\right)$ and " $\Gamma \cap B_{N} \neq \varnothing$ " $\left(H_{m}\right)$ can be made using the test of randomness which is described in Section 2.3. If the hypothesis $H_{m}$ is accepted, the center of the current window is marked as an edge point. Repeating this process for each position of the window, we find all edge points.

Numerical results of an application of the above algorithm are illustrated by the following example. Fig. 1 represents a synthetic image of square and circle edges 
FIG. 1. A $101 \times 101$ synthetic image of square and circle step edges corrupted by noise.

with the jump magnitude $D=1.5$ specified at a square $101 \times 101$ grid. The image is corrupted by noise with the uniform distribution and standard deviation $\sigma=0.75$. The window size has been chosen $N=7$, the probability of false alarm has been $\epsilon=0.01$. Fig. 2 represents the image of detected edges of Fig. 1.

\section{Consistency against trend in location}

\subsection{One-Dimensional CASE, EQUisPaCED DESign MOdel}

Let $\left\{x_{k}\right\}_{k=1}^{N}$ be a random sequence of size $N$. The problem is to test the null hypothesis

$$
H_{0}: \quad F_{1}(x)=F_{2}(x)=\cdots=F_{N}(x),
$$

where $F_{k}(x)$ is a continuous distribution function of the random variable $x_{k}, k=$ $1, \ldots, N$, against the alternative

$$
H_{1}: \quad F_{k}(x)=F\left(x-\theta_{k}\right), k=1, \ldots, N,
$$

where $F$ is a continuously differentiable distribution function with $f(x):=F^{\prime}(x)$, $\sup _{x \in \mathbb{R}^{1}} f(x)<\infty$, and $\theta_{k} \in \mathbb{R}^{1}$ are some constants that are not all equal. Let $R_{i}$ be the rank of the element $x_{i}, i=1, \ldots, N$. The test criterion is statistic (2.1.4):

$$
\nu_{N}:=\frac{1}{N-1} \sum_{k=1}^{N-1}\left(\frac{R_{k+1}-R_{k}}{N}\right)^{2} .
$$


FIG. 2. Detected step edges of Fig. 1.

We have $\nu_{N} \stackrel{\mathrm{ms}}{\longrightarrow} 1 / 6$ as $N \rightarrow \infty$ if $H_{0}$ is true [see Sec. 2.1, cf. also WW]. As in Sections 2.1 and 2.2, to prove consistency of the test against the alternative $H_{1}$, it is sufficient to prove that $\nu_{N}$ converges to some constant $E_{\infty}$ as $N \rightarrow \infty$ if $H_{1}$ holds, and that $E_{\infty}<1 / 6$. Let us study the asymptotic behavior of $\nu_{N}$ as $N \rightarrow \infty$ under $H_{1}$. Suppose that the trend $\theta_{k}, k=1, \ldots, N$, satisfies the condition:

$$
\phi(t):=\lim _{N \rightarrow \infty, k / N \rightarrow t} \theta_{k}, \quad 0 \leq t \leq 1 ; \quad \phi(t) \in C[0,1], \phi(t) \not \equiv \text { const. }
$$

Fix $m \geq 2$ and define the intervals $\Delta_{l}$

$$
\Delta_{l}:=[(l-1) / m, l / m), l=1, \ldots, m .
$$

Let $\nu_{N}^{(m)}$ be statistic (3.1.3) calculated in the case when the trend $\tilde{\theta}_{k}, k=1, \ldots, N$, is constant inside each interval $\Delta_{l}$ :

$$
\tilde{\theta}_{k}=\phi(l / m) \text { for } l \text { such that } k / N \in \Delta_{l}, k=1, \ldots, N .
$$

Using the results obtained in Sec. 2.2, we have

$$
\nu_{N}^{(m)} \underset{N \rightarrow \infty}{\stackrel{\mathrm{ms}}{\longrightarrow}} 2\left\{\frac{1}{3}-\frac{1}{m} \sum_{l=1}^{m}\left(\int_{\mathbb{R}} G^{(m)}(x) g_{l}^{(m)}(x) d x\right)^{2}\right\}:=E_{m}<1 / 6,
$$




$$
G^{(m)}(x)=\frac{1}{m} \sum_{l=1}^{m} F(x-\phi(l / m)), \quad g_{l}^{(m)}(x)=f(x-\phi(l / m)) .
$$

Denote $E_{\infty}:=\lim _{m \rightarrow \infty} E_{m}$. Existence of the limit $E_{\infty}$ can be established, an analytical expression for this limit is given in formula (3.1.15). Let " $\stackrel{p}{\longrightarrow}$ " denote convergence in probability.

Theorem 3.1. Under assumptions (3.1.2) and (3.1.4) we have $\nu_{N} \underset{N \rightarrow \infty}{\stackrel{\mathrm{p}}{\longrightarrow}} E_{\infty}$.

Let $\left\{y_{k}\right\}_{k=1}^{N}$ be a random sample from the distribution $F(x)$, and let us define two other sequences $\left\{\hat{y}_{k}:=y_{k}+\theta_{k}\right\}_{k=1}^{N},\left\{\hat{y}_{k}^{(m)}:=y_{k}+\tilde{\theta}_{k}\right\}_{k=1}^{N}$. Let $\hat{R}_{k}, \hat{R}_{k}^{(m)}$ be the ranks of the elements $\hat{y}_{k}, \hat{y}_{k}^{(m)}$, respectively. Denote $\hat{r}_{k}=\hat{R}_{k} / N, \hat{r}_{k}^{(m)}=$ $\hat{R}_{k}^{(m)} / N$, and let $\hat{\nu}_{N}, \hat{\nu}_{N}^{(m)}$ be the values of statistic (3.1.3) calculated for sequences $\left\{\hat{y}_{k}\right\}_{k=1}^{N},\left\{\hat{y}_{k}^{(m)}\right\}_{k=1}^{N}$, respectively. First, we prove two auxiliary lemmas.

Lemma 3.1. For every $\epsilon>0$ there exists $M_{\epsilon}$ such that $\lim _{N \rightarrow \infty} P\left\{\left|\hat{\nu}_{N}-\hat{\nu}_{N}^{\left(M_{\epsilon}\right)}\right| \geq\right.$ $\epsilon\}=0$.

Proof of Lemma 3.1. Fix $\epsilon>0$, denote $h_{m}:=\max _{1 \leq k \leq N}\left|\theta_{k}-\tilde{\theta}_{k}\right|$, where $m$ is the same as in (3.1.5), and find $M_{\epsilon}$ such that

$$
\sup _{x}\left|F\left(x+2 h_{M_{\epsilon}}\right)-F\left(x-2 h_{M_{\epsilon}}\right)\right|<\epsilon / 24 .
$$

Existence of such $M_{\epsilon}$ follows the uniform boundedness of $f(x)$ and (3.1.4)-(3.1.6). Denote for brevity in what follows $\hat{\nu}_{N}^{(\epsilon)}:=\hat{\nu}_{N}^{\left(M_{\epsilon}\right)}, \hat{r}_{k}^{(\epsilon)}:=\hat{r}_{k}^{\left(M_{\epsilon}\right)}, h_{\epsilon}:=h_{M_{\epsilon}}$. ¿From the definitions of $\hat{\nu}_{N}, \hat{\nu}_{N}^{(\epsilon)}$ we obtain:

$$
\begin{aligned}
\left|\hat{\nu}_{N}-\hat{\nu}_{N}^{(\epsilon)}\right| & =\left|\frac{1}{N-1} \sum_{k=1}^{N-1}\left[\left(\hat{r}_{k+1}-\hat{r}_{k}\right)^{2}-\left(\hat{r}_{k+1}^{(\epsilon)}-\hat{r}_{k}^{(\epsilon)}\right)^{2}\right]\right| \\
& \leq \frac{4}{N-1} \sum_{k=1}^{N-1}\left|\left(\hat{r}_{k+1}-\hat{r}_{k+1}^{(\epsilon)}\right)-\left(\hat{r}_{k}-\hat{r}_{k}^{(\epsilon)}\right)\right| \leq \frac{8}{N-1} \sum_{k=1}^{N}\left|\hat{r}_{k}-\hat{r}_{k}^{(\epsilon)}\right|
\end{aligned}
$$

Let us estimate the probability $P\left\{\left|\hat{r}_{k}-\hat{r}_{k}^{(\epsilon)}\right|>\epsilon\right\}$. ¿From the definition of $h_{\epsilon}$ we have

$$
P\left\{\left|\hat{r}_{k}-\hat{r}_{k}^{(\epsilon)}\right|>\epsilon\right\} \leq P\left\{\frac{\#\left\{l: y_{l}+\tilde{\theta}_{l} \in\left[y_{k}+\tilde{\theta}_{k}-2 h_{\epsilon}, y_{k}+\tilde{\theta}_{k}+2 h_{\epsilon}\right]\right\}}{N}>\epsilon\right\} .
$$

Similarly,

$$
P\left\{\frac{1}{N} \sum_{k=1}^{N}\left|\hat{r}_{k}-\hat{r}_{k}^{(\epsilon)}\right|>\epsilon\right\} \leq P\left\{\sup _{y \in \mathbb{R}} \frac{\#\left\{k: y_{k}+\tilde{\theta}_{k} \in\left[y-2 h_{\epsilon}, y+2 h_{\epsilon}\right]\right\}}{N}>\epsilon\right\} .
$$


Together with (3.1.9), this yields

$$
\begin{gathered}
P\left\{\left|\hat{\nu}_{N}-\hat{\nu}_{N}^{(\epsilon)}\right|>\epsilon\right\} \leq P\left\{\sup _{y \in \mathbb{R}} \frac{\#\left\{k: y_{k}+\tilde{\theta}_{k} \in\left[y-2 h_{\epsilon}, y+2 h_{\epsilon}\right]\right\}}{N}>\tilde{\epsilon} / 8\right\} \leq \\
P\left\{\sup _{y \in \mathbb{R}} \frac{\sum_{l=1}^{M_{\epsilon}} \#\left\{k: k / N \in \Delta_{l}, y_{k}+\phi\left(l / M_{\epsilon}\right) \in\left[y-2 h_{\epsilon}, y+2 h_{\epsilon}\right]\right\}}{N}>\tilde{\epsilon} / 8\right\} \leq \\
\sum_{l=1}^{M_{\epsilon}} P\left\{\sup _{y \in \mathbb{R}} \frac{\#\left\{k: k / N \in \Delta_{l}, y_{k} \in\left[y-2 h_{\epsilon}, y+2 h_{\epsilon}\right]\right\}}{N / M_{\epsilon}}>\tilde{\epsilon} / 8\right\}= \\
\sum_{l=1}^{M_{\epsilon}} P\left\{\sup _{y \in \mathbb{R}}\left(F_{l}\left(y+2 h_{\epsilon}\right)-F_{l}\left(y-2 h_{\epsilon}\right)\right)>\tilde{\epsilon} / 8\right\},
\end{gathered}
$$

where $\tilde{\epsilon}=\epsilon(N-1) / N$, and $F_{l}(y)$ is the empirical distribution function of the sample $\left\{y_{k}\right\}_{k \in \Delta_{l}}, 1 \leq l \leq M_{\epsilon}$. In (3.1.11), we used the inequality $P\left\{\xi_{1}+\cdots+\xi_{M}>M \epsilon\right\} \leq$ $P\left\{\xi_{1}>\epsilon\right\}+\cdots+P\left\{\xi_{M}>\epsilon\right\}$, where $\xi_{i}$ are arbitrary random variables. From (3.1.8) and (3.1.11) we obtain

$$
\begin{array}{r}
P\left\{\left|\hat{\nu}_{N}-\hat{\nu}_{N}^{(\epsilon)}\right|>\epsilon\right\} \leq \sum_{l=1}^{M_{\epsilon}} P\left\{\sup _{y \in \mathbb{R}}\left|F_{l}\left(y+2 h_{\epsilon}\right)-F\left(y+2 h_{\epsilon}\right)\right|\right. \\
\quad+\sup _{y \in \mathbb{R}}\left|F\left(y+2 h_{\epsilon}\right)-F\left(y-2 h_{\epsilon}\right)\right| \\
\left.\quad+\sup _{y \in \mathbb{R}}\left|F_{l}\left(y-2 h_{\epsilon}\right)-F\left(y-2 h_{\epsilon}\right)\right|>\tilde{\epsilon} / 8\right\} \\
\leq \sum_{l=1}^{M_{\epsilon}} P\left\{\sup _{y \in \mathbb{R}}\left|F_{l}(y)-F(y)\right|>\epsilon(1-3 /(2 N)) / 24\right\} .
\end{array}
$$

Taking the limit as $N \rightarrow \infty$ on both sides of the last inequality and using the Glivenko theorem, we complete the proof of Lemma 3.1.

Lemma 3.2. Fix an arbitrary $\epsilon>0$ and find $M_{\epsilon}$ such that (3.1.8) holds. Then for any $a \notin\left[E_{M_{\epsilon}}-\epsilon, E_{M_{\epsilon}}+\epsilon\right]$, where $E_{M_{\epsilon}}$ is the same as in (3.1.7a) with $m=M_{\epsilon}$, the following equation holds

$$
\lim _{N \rightarrow \infty}\left|P\left\{\nu_{N}<a\right\}-P\left\{\nu_{N}^{\left(M_{\epsilon}\right)}<a\right\}\right|=0 .
$$

Proof of Lemma 3.2. Denote $\nu_{N}^{(\epsilon)}:=\nu_{N}^{\left(M_{\epsilon}\right)}$ and write the dependence of $\hat{\nu}_{N}$ and $\hat{\nu}_{N}^{(\epsilon)}$ on $y_{1}, \ldots, y_{N}$ implicitly as $\hat{\nu}_{N}=\hat{\nu}_{N}(y)$ and $\hat{\nu}_{N}^{(\epsilon)}=\hat{\nu}_{N}^{(\epsilon)}(y)$. From the definitions of $\nu_{N}, \nu_{N}^{(\epsilon)}, \hat{\nu}_{N}$, and $\hat{\nu}_{N}^{(\epsilon)}$, we obtain

$$
\begin{gathered}
\left|P\left\{\nu_{N}<a\right\}-P\left\{\nu_{N}^{(\epsilon)}<a\right\}\right|=\mid \int \theta\left(a-\hat{\nu}_{N}(y)\right) f_{N}(y) d y- \\
\int \theta\left(a-\hat{\nu}_{N}^{(\epsilon)}(y)\right) f_{N}(y) d y\left|\leq \int\right| \theta\left(a-\hat{\nu}_{N}(y)\right)-\theta\left(a-\hat{\nu}_{N}^{(\epsilon)}(y)\right) \mid f_{N}(y) d y
\end{gathered}
$$


where $\int:=\int_{\mathbb{R}^{N}}, d y:=d y_{1} \ldots d y_{N}, y:=y_{1} \ldots y_{N}, f_{N}(y):=f\left(y_{1}\right) \ldots f\left(y_{N}\right)$, and

$$
\theta(t)= \begin{cases}1, & t>0 \\ 0, & t<0\end{cases}
$$

The integral on the right-hand side of (3.1.12) can be estimated as follows

$$
\begin{gathered}
\int\left|\theta\left(a-\hat{\nu}_{N}(y)\right)-\theta\left(a-\hat{\nu}_{N}^{(\epsilon)}(y)\right)\right| f_{N}(y) d y \leq \int_{\left\{y:\left|\hat{\nu}_{N}(y)-\hat{\nu}_{N}^{(\epsilon)}(y)\right|<\epsilon\right\}} \mid \theta\left(a-\hat{\nu}_{N}(y)\right)- \\
\theta\left(a-\hat{\nu}_{N}^{(\epsilon)}(y)\right) \mid f_{N}(y) d y+P\left\{\left|\hat{\nu}_{N}-\hat{\nu}_{N}^{(\epsilon)}\right|>\epsilon\right\}=\int_{\substack{\left\{y:\left|\hat{\nu}_{N}^{(\epsilon)}(y)-a\right|<\epsilon,\left|\hat{\nu}_{N}(y)-\hat{\nu}_{N}^{(\epsilon)}(y)\right|<\epsilon\right\}}} 1 \cdot f_{N}(y) d y+ \\
P\left\{\left|\hat{\nu}_{N}-\hat{\nu}_{N}^{(\epsilon)}\right|>\epsilon\right\} \leq P\left\{\left|\hat{\nu}_{N}^{(\epsilon)}-a\right|<\epsilon\right\}+P\left\{\left|\hat{\nu}_{N}-\hat{\nu}_{N}^{(\epsilon)}\right|>\epsilon\right\} .
\end{gathered}
$$

¿From (3.1.7a) we have $\lim _{N \rightarrow \infty} P\left\{\left|\hat{\nu}_{N}^{(\epsilon)}-a\right|<\epsilon\right\}=0$ for $a \notin\left[E_{M_{\epsilon}}-\epsilon, E_{M_{\epsilon}}+\epsilon\right]$, and from Lemma 3.1 we have $\lim _{N \rightarrow \infty} P\left\{\left|\hat{\nu}_{N}-\hat{\nu}_{N}^{(\epsilon)}\right|>\epsilon\right\}=0$. This together with (3.1.12) and (3.1.13) proves Lemma 3.2.

Proof of Theorem 3.1. Pick an arbitrary $\epsilon>0$ and find $M_{\epsilon}$ satisfying (3.1.8) such that $\left|E_{M_{\epsilon}}-E_{\infty}\right|<\epsilon$. Thus, using Lemma 3.2 and (3.1.7a), we get

$$
\lim _{N \rightarrow \infty} P\left\{\nu_{N}<E_{\infty}-2 \epsilon\right\}=\lim _{N \rightarrow \infty} P\left\{\nu_{N}^{(\epsilon)}<E_{\infty}-2 \epsilon\right\}=0 .
$$

Similarly,

$$
\lim _{N \rightarrow \infty} P\left\{\nu_{N}<E_{\infty}+2 \epsilon\right\}=\lim _{N \rightarrow \infty} P\left\{\nu_{N}^{(\epsilon)}<E_{\infty}+2 \epsilon\right\}=1 .
$$

Combining (3.1.14a) and (3.1.14b) proves Theorem 3.1.

Now we prove that the statistic $\nu_{N}$ can be used for testing $H_{0}$ against $H_{1}$ for an arbitrary trend satisfying (3.1.4), where $\phi(t) \not \equiv$ const is an arbitrary continuous function.

Theorem 3.2. Under assumptions (3.1.2) and (3.1.4) we have $E_{\infty}<1 / 6$.

¿From (3.1.4) - (3.1.7a) it follows that

$$
\begin{gathered}
G^{(m)}(x) \underset{m \rightarrow \infty}{\longrightarrow} \int_{0}^{1} F(x-\phi(t)) d t \\
E_{\infty}:=\lim _{m \rightarrow \infty} E_{m}=2\left\{\frac{1}{3}-\int_{0}^{1}\left(\int_{\mathbb{R}}^{1}\left[\int_{0}^{1} F(x-\phi(t)) d t\right] f(x-\phi(s)) d x\right)^{2} d s\right\} .
\end{gathered}
$$

Thus we need to prove the inequality

$$
I(\phi):=\int_{0}^{1}\left\{\int_{\mathbb{R}}\left[\int_{0}^{1} F(x-\phi(t)) d t\right] f(x-\phi(s)) d x\right\}^{2} d s>\frac{1}{4} .
$$


Let us prove (3.1.16). Denote $a:=\min _{t \in[0,1]} \phi(t), \quad b:=\max _{t \in[0,1]} \phi(t)$. Since $\phi(t) \not \equiv$ const, we have $a<b$. Pick an arbitrary $y$ such that $a<y<b$, denote $I_{y}:=\{t: t \in[0,1], \phi(t) \geq y\}$, and let $\psi(t)$ be any locally integrable nonnegative function, $\psi(t) \not \equiv 0, \operatorname{supp} \psi(t) \subseteq I_{y}$. Denote also

$$
\tilde{\phi}_{y}(t):=\tilde{\phi}(t):= \begin{cases}\phi(t), & t \notin I_{y}, \\ y, & t \in I_{y}\end{cases}
$$

First we prove an auxiliary lemma, then the proof of Theorem 3.2 is given.

Lemma 3.3. One has

$$
\left.\frac{\partial}{\partial \alpha} I(\tilde{\phi}+\alpha \psi)\right|_{\alpha=0}>0
$$

Proof of Lemma 3.3. Changing variables $x^{\prime}=x-\phi(s)$ and differentiating $I(\tilde{\phi}+\alpha \psi)$ with respect to $\alpha$, we get

$$
\begin{aligned}
\left.\frac{\partial I}{\partial \alpha}\right|_{\alpha=0}=2 \int_{0}^{1}\left\{\int_{0}^{1} \int_{\mathbb{R}} F(x+\tilde{\phi}(s)-\tilde{\phi}(t)) f(x) d x d t\right. \\
\left.\quad \times \int_{0}^{1} \int_{\mathbb{R}} f(y+\tilde{\phi}(s)-\tilde{\phi}(\tau)) f(y) d y(\psi(s)-\psi(\tau)) d \tau\right\} d s .
\end{aligned}
$$

Note that the above change of variables makes it clear that $I(\phi)$ is Gateaux differentiable in $C[0,1]$. This observation will be used later. Since $\psi(t)$ vanishes outside $I_{y}$ and $\tilde{\phi}(t)=y$ inside $I_{y}$, we may write

$$
\begin{aligned}
\left.\frac{\partial I}{\partial \alpha}\right|_{\alpha=0}=2\left\{\int_{0}^{1} A(y-\tilde{\phi}(t)) d t \int_{0}^{1} B(y-\tilde{\phi}(\tau)) d \tau \int_{I_{y}} \psi(s) d s\right. \\
\left.-\int_{0}^{1} \int_{0}^{1} A(\tilde{\phi}(s)-\tilde{\phi}(t)) d t B(\tilde{\phi}(s)-y) d s \int_{I_{y}} \psi(\tau) d \tau\right\},
\end{aligned}
$$

where

$$
A(u):=\int_{\mathbb{R}} F(x+u) f(x) d x, \quad B(u):=\int_{\mathbb{R}} f(x+u) f(x) d x .
$$

Since $\int_{I_{y}} \psi(t) d t>0$, we see that the assertion of Lemma 3.3 is equivalent to

$$
\int_{0}^{1} A(y-\tilde{\phi}(t)) d t \int_{0}^{1} B(y-\tilde{\phi}(\tau)) d \tau>\int_{0}^{1} \int_{0}^{1} A(\tilde{\phi}(s)-\tilde{\phi}(t)) d t B(\tilde{\phi}(s)-y) d s .
$$

Since $B(u)$ is an even function (see (3.1.19)), we can rewrite the last inequality as

$$
\int_{0}^{1} \int_{0}^{1} B(\tilde{\phi}(s)-y)\{A(y-\tilde{\phi}(t))-A(\tilde{\phi}(s)-\tilde{\phi}(t))\} d t d s>0 .
$$


Since $B(u) \geq 0, A(u)$ is a nondecreasing function, and $\tilde{\phi}(s) \leq y$, inequality (3.1.20) is established with " $\geq$ " in place of " $>$ ". Now let us prove the strict inequality (3.1.20). First, consider the integral over $s$. Clearly, there exists an $s_{0}$ such that $\left|y-\tilde{\phi}\left(s_{0}\right)\right| \ll 1, y>\tilde{\phi}\left(s_{0}\right)$ and $B\left(y-\tilde{\phi}\left(s_{0}\right)\right)>0$, because $B(0)=\int_{\mathbb{R}} f^{2}(x) d x>0$. Fix $s, t=s_{0}$ in the expression in braces in (3.1.20). From (3.1.19) we obtain

$$
\begin{aligned}
A\left(y-\tilde{\phi}\left(s_{0}\right)\right)-A(0) & =\int_{-\infty}^{\infty}\left[F\left(x+\left(y-\tilde{\phi}\left(s_{0}\right)\right)\right)-F(x)\right] f(x) d x \\
& =\int_{-\infty}^{\infty} F\left(x+\left(y-\tilde{\phi}\left(s_{0}\right)\right)\right) f(x) d x-\frac{1}{2}>0,
\end{aligned}
$$

since $F(x)$ is nondecreasing, continuously differentiable, has points of growth, and $y-\tilde{\phi}\left(s_{0}\right)>0$. Thus the integrand in (3.1.20) is strictly positive in a neighborhood of the point $\left(s_{0}, s_{0}\right)$. This together with continuity of $A(u)$ and $B(u)$ proves Lemma 3.3 .

Proof of Theorem 3.2. Fix $n \geq 2$ and consider a partition of the interval $[a, b]: a=$ $y_{1}<y_{2}<\cdots<y_{n+1}=b$, with $y_{k+1}-y_{k}=h, k=1, \ldots, n, h=(b-a) / n$. Define functions

$$
\psi_{y_{l}}(t)=\left\{\begin{array}{ll}
1, & \phi(t) \geq y_{l}, \\
0, & \phi(t)<y_{l}
\end{array}, \phi_{n}(t)=a+h \sum_{l=1}^{n} \psi_{y_{l}}(t), \phi_{n, k}(t)=a+h \sum_{l=1}^{k} \psi_{y_{l}}(t),\right.
$$

$k=1, \ldots, n$. Clearly, we have $I\left(\phi_{n, k+1}\right)=I\left(\phi_{n, k}\right)+\left.\frac{\partial I\left(\phi_{n, k}+\alpha \psi_{y_{k}}\right)}{\partial \alpha}\right|_{\alpha=0} h+o(h)$. Thus, we get

$$
I\left(\phi_{n}\right)=\frac{1}{4}+\left.h \sum_{k=1}^{n} \frac{\partial I\left(\phi_{n, k}+\alpha \psi_{y_{k}}\right)}{\partial \alpha}\right|_{\alpha=0}+o(1)
$$

where we have used the equation $I$ (const) $=1 / 4$, which follows from definition (3.1.16). Note that $\max _{t \in[0,1]}\left|\phi(t)-\phi_{n}(t)\right| \rightarrow 0, \max _{t \in[0,1]}\left|\tilde{\phi}_{y}(t)-\phi_{n,[y n]}(t)\right| \rightarrow 0$ as $n \rightarrow \infty$, where the function $\tilde{\phi}_{y}$ was defined in (3.1.17), and $[u]$ is the integer part of $u$. Using this and the continuity of the functional $I(\phi)$ and its Gateaux derivative in the space $C[0,1]$, we obtain from (3.1.22), by taking $n \rightarrow \infty(h \rightarrow 0)$, the following formula:

$$
I(\phi)=\frac{1}{4}+\left.\int_{a}^{b} \frac{\partial I\left(\tilde{\phi}_{y}+\alpha \psi_{y}\right)}{\partial \alpha}\right|_{\alpha=0} d y
$$

Using Lemma 3.3, we see that the integrand in (3.1.23) is strictly positive for all $y, a<y<b$. Therefore $I(\phi)>1 / 4$, and Theorem 3.2 is proved.

\subsection{Multidimensional Case, Regular Design MOdel}

Let the given data be $x_{k_{1}, \ldots, k_{d}}, 1 \leq k_{i} \leq \beta_{i} N, 0<\beta_{i}<\infty, i=1, \ldots, d$. Without loss of generality we may assume $\beta_{i}$ to be integers. Denote $k:=\left(k_{1}, \ldots, k_{d}\right) \in$ 
$\mathbb{N}^{d}, t:=\left(t_{1}, \ldots, t_{d}\right) \in \mathbb{R}^{d}, B_{N}:=\left\{k: 1 \leq k_{i} \leq \beta_{i} N, i=1, \ldots, d\right\}, D:=\{t: 0 \leq$ $\left.t_{i} \leq \beta_{i}, i=1, \ldots, d\right\}$. The problem is to test the null hypothesis

$$
H_{0}: \quad F_{k}(x)=F_{j}(x) \quad \forall k, j \in B_{N}
$$

against the alternative

$$
H_{1}: \quad F_{k}(x)=F\left(x-\theta_{k}\right), k \in B_{N},
$$

where $F$ is a continuously differentiable distribution function with $f(x):=F^{\prime}(x)$, $\sup _{x \in \mathbb{R}^{1}} f(x)<\infty$, and $\theta_{k} \in \mathbb{R}^{1}$ are some constants satisfying the condition:

$$
\phi(t):=\lim _{N \rightarrow \infty, k / N \rightarrow t} \theta_{k}, t \in D ; \quad \phi(t) \in C[D], \phi(t) \not \equiv \text { const. }
$$

As in Section 2.3, for an arbitrary multiindex $k \in B_{N}$ we define the set of multiindices neighboring to $k$ by the formula $L(k):=\left\{l \in B_{N}: l \neq k, \max _{1 \leq i \leq d} \mid l_{i}-\right.$ $\left.k_{i} \mid=1\right\}$. The test criterion is statistic (2.3.3):

$$
\nu_{N}:=\frac{1}{M_{N}} \sum_{k \in B_{N}} \sum_{l \in L(k)}\left(\frac{R_{k}-R_{l}}{\hat{N}}\right)^{2},
$$

where $\hat{N}:=\beta_{1} \ldots \beta_{d} N^{d}$, and $M_{N}$ is the number of elements in double sum (3.2.4). We have $\nu_{N} \stackrel{\mathrm{ms}}{\longrightarrow} 1 / 6$ as $N \rightarrow \infty$ if $H_{0}$ is true [see Sec. 2.3, cf. also CO]. Similarly to the one-dimensional case, to prove consistency of the test against the alternative $H_{1}$, it is sufficient to prove that $\nu_{N}$ converges to some constant $E_{\infty}$ as $N \rightarrow \infty$ if $H_{1}$ holds, and that $E_{\infty}<1 / 6$. Let us study the asymptotic behavior of $\nu_{N}$ as $N \rightarrow \infty$ under $H_{1}$. Fix $m \geq 2$ and consider the following sublattices

$$
\begin{gathered}
\Delta_{l}:=\left[\beta_{1} \frac{l_{1}-1}{m}, \beta_{1} \frac{l_{1}}{m}\right) \times \cdots \times\left[\beta_{d} \frac{l_{d}-1}{m}, \beta_{d} \frac{l_{d}}{m}\right), \\
l:=\left(l_{1}, \ldots, l_{d}\right) \in \mathbb{N}^{d}, 1 \leq l_{i} \leq m, 1 \leq i \leq d .
\end{gathered}
$$

Let $\nu_{N}^{(m)}$ be statistic (3.2.4) calculated in the case when the trend $\tilde{\theta}_{k}, k \in B_{N}$, is constant inside each sublattice $\Delta_{l}$

$$
\tilde{\theta}_{k}=\phi(l / m) \text { for } k / N \in \Delta_{l}, 1 \leq l_{i} \leq m, 1 \leq i \leq d .
$$

Using the results obtained in Section 2.2, we have

$$
\begin{gathered}
\nu_{N}^{(m)} \underset{N \rightarrow \infty}{\stackrel{\mathrm{ms}}{\longrightarrow}} 2\left\{\frac{1}{3}-\frac{1}{m^{d}} \sum_{i=1}^{d} \sum_{l_{i}=1}^{m}\left(\int_{\mathbb{R}} G^{(m)}(x) g_{l}^{(m)}(x) d x\right)^{2}\right\}:=E_{m}<1 / 6, \\
G^{(m)}(x)=\frac{1}{m^{d}} \sum_{i=1}^{d} \sum_{l_{i}=1}^{m} F(x-\phi(l / m)), \quad g_{l}^{(m)}(x)=f(x-\phi(l / m)) .
\end{gathered}
$$

Denoting $d t:=d t_{1} \ldots d t_{d}, d s:=d s_{1} \ldots d s_{d}$ and taking the limit as $m \rightarrow \infty$, we obtain

$$
E_{\infty}:=\lim _{m \rightarrow \infty} E_{m}=2\left\{\frac{1}{3}-\int_{D}\left(\int_{\mathbb{R}}\left[\int_{D} F(x-\phi(t)) d t\right] f(x-\phi(s)) d x\right)^{2} d s\right\} .
$$

Theorem 3.1'. Under assumptions (3.2.2) and (3.2.3) we have $\nu_{N} \underset{N \rightarrow \infty}{\stackrel{\mathrm{p}}{\longrightarrow}} E_{\infty}$.

Theorem 3.2'. Under assumptions (3.2.2) and (3.2.3) we have $E_{\infty}<1 / 6$.

The proofs of Theorems $3.1^{\prime}$ and $3.2^{\prime}$ are omitted because they are similar to those of Theorems 3.1 and 3.2. 


\subsection{RANDOM DESIGN MODEL}

Let the observation points $\left\{\rho_{k}\right\}_{k=1}^{N}$ be randomly chosen inside an open bounded domain $D \subset \mathbb{R}^{d}, d \geq 1$, and let $\left\{x_{k}\right\}_{k=1}^{N}$ be a set of corresponding observations. The problem is to test the null hypothesis

$$
H_{0}: \quad F_{1}(x)=F_{2}(x)=\cdots=F_{N}(x),
$$

where $F_{k}(x)$ is a continuous distribution function of the random variable observed at the point $\rho_{k}, k=1, \ldots, N$, against the alternative

$$
H_{1}: \quad F_{k}(x)=F\left(x-\phi\left(\rho_{k}\right)\right), \phi(t) \in C[\bar{D}], \phi(t) \not \equiv \text { const },
$$

where $F$ is a continuously differentiable distribution function with $f(x):=F^{\prime}(x)$, $\sup _{x \in \mathbb{R}^{1}} f(x)<\infty$, and $\bar{D}$ is the closure of $D$. The test criterion is statistic (2.4.3):

$$
\nu_{N}:=\frac{1}{N} \sum_{k=1}^{N}\left(\frac{R_{n(k)}-R_{k}}{N}\right)^{2}
$$

where $n(k)$ is the index of the point closest to $\rho_{k},\left|\rho_{n(k)}-\rho_{k}\right|=\min _{\substack{\leq j \leq N \\ j \neq k}}\left|\rho_{j}-\rho_{k}\right|$.

Note that $n(k)$ is unique with probability 1 . As in previous sections, $\nu_{N} \stackrel{\mathrm{ms}}{\longrightarrow} 1 / 6$ as $N \rightarrow \infty$ if $H_{0}$ is true (cf. Sec. 2.4). Therefore, to prove consistency of the test against the alternative $H_{1}$, it is sufficient to prove that $\nu_{N}$ converges to some constant $E_{\infty}$ as $N \rightarrow \infty$ if $H_{1}$ holds, and that $E_{\infty}<1 / 6$. Let us study the asymptotic behavior of $\nu_{N}$ as $N \rightarrow \infty$ under $H_{1}$. Fix $m \geq 2$ and consider a finite, disjoint, measurable partition of $D: \bigcup_{j=1}^{m} D_{j}=D, D_{i} \cap D_{j}=\varnothing, i \neq j, \lambda\left(D_{j}\right)=$ $\lambda(D) / m, j=1, \ldots, m$, where $\lambda(D)$ is the Lebesgue measure in $\mathbb{R}^{d}$. Fix arbitrarily points $t_{j} \in D_{j}$ and define a piecewise-constant trend function $\tilde{\phi}(t):=\phi\left(t_{j}\right)$ if $t \in D_{j}$. Let $\nu_{N}^{(m)}$ be statistic (3.3.3) calculated for such a trend. Using the results obtained in Section 2.3, we have

$$
\begin{gathered}
\nu_{N}^{(m)} \underset{N \rightarrow \infty}{\stackrel{\mathrm{ms}}{\longrightarrow}} 2\left\{\frac{1}{3}-\frac{1}{m} \sum_{i=1}^{m}\left(\int_{\mathbb{R}} G^{(m)}(x) g_{i}^{(m)}(x) d x\right)^{2}\right\}:=E_{m}<1 / 6, \\
G^{(m)}(x)=\frac{1}{m} \sum_{i=1}^{m} F\left(x-\phi\left(t_{i}\right)\right), \quad g_{i}^{(m)}(x)=f\left(x-\phi\left(t_{i}\right)\right) .
\end{gathered}
$$

As in Section 3.2, we get

$$
E_{\infty}:=\lim _{m \rightarrow \infty} E_{m}=2\left\{\frac{1}{3}-\int_{D}\left(\int_{\mathbb{R}}\left[\int_{D} F(x-\phi(t)) d t\right] f(x-\phi(s)) d x\right)^{2} d s\right\} .
$$

Theorem 3.1". Under assumptions (3.3.2) we have $\nu_{N} \underset{N \rightarrow \infty}{\stackrel{\mathrm{p}}{\longrightarrow}} E_{\infty}$.

Proof. Since the proofs of Theorems 3.1 and $3.1^{\prime \prime}$ are similar, we present here only a brief discussion of differences between them. 
Let $\left\{\rho_{k}\right\}_{k=1}^{N}$ be a set of random observation points and let $\left\{y_{k}\right\}_{k=1}^{N}$ be a random sample from the distribution $F(x)$. As in Section 3.1, we define two other sequences $\left\{\hat{y}_{k}:=y_{k}+\phi\left(\rho_{k}\right)\right\}_{k=1}^{N},\left\{\hat{y}_{k}^{(m)}:=y_{k}+\tilde{\phi}\left(\rho_{k}\right)\right\}_{k=1}^{N}$. Thus, the statistics $\hat{\nu}_{N}$ and $\hat{\nu}_{N}^{(m)}$ calculated for sequences $\left\{\hat{y}_{k}\right\}_{k=1}^{N}$ and $\left\{\hat{y}_{k}^{(m)}\right\}_{k=1}^{N}$, respectively, depend jointly on two random sets $\rho:=\left\{\rho_{k}\right\}_{k=1}^{N}$ and $\left\{y_{k}\right\}_{k=1}^{N}$. It is not hard to see that Lemma 3.1

still holds for such $\hat{\nu}_{N}$ and $\hat{\nu}_{N}^{(m)}$. The differences between the proofs are

a) in the definition of $h_{m}$ (see above (3.1.8)), here it should be

$$
h_{m}:=\max _{t \in D}|\phi(t)-\tilde{\phi}(t)|
$$

and

b) in the fact that each set $D_{i}$ contains now a random number of observation points.

Since the number of observation points inside each $D_{i}$ goes to infinity with probability 1 as $N \rightarrow \infty$, we conclude that the empirical distribution functions inside each $D_{i}$ converge to $F(x)$ with probability 1 and the conclusion of Lemma 3.1 follows.

Lemma 3.2 also holds in this case. Indeed, the inequality

$$
\left|P\left\{\nu_{N}<a\right\}-P\left\{\nu_{N}^{(\epsilon)}<a\right\}\right| \leq P\left\{\left|\hat{\nu}_{N}^{(\epsilon)}-a\right|<\epsilon\right\}+P\left\{\left|\hat{\nu}_{N}-\hat{\nu}_{N}^{(\epsilon)}\right|>\epsilon\right\}
$$

easily follows if we combine (3.1.12) and (3.1.13) and write the resulting inequality using the notation of this section as

$$
\left|P\left\{\nu_{N}<a \mid \rho\right\}-P\left\{\nu_{N}^{(\epsilon)}<a \mid \rho\right\}\right| \leq P\left\{\left|\hat{\nu}_{N}^{(\epsilon)}-a\right|<\epsilon \mid \rho\right\}+P\left\{\left|\hat{\nu}_{N}-\hat{\nu}_{N}^{(\epsilon)}\right|>\epsilon \mid \rho\right\} .
$$

The rest of the argument goes without changes.

Theorem 3.2". Under assumptions (3.3.2) one has $E_{\infty}<1 / 6$.

The proof of Theorem $3.2^{\prime \prime}$ is the same as that of Theorem 3.2.

\section{REFERENCES}

[A] R. J. Aiyar, Asymptotic efficiency of rank tests of randomness against autocorrelation, Annals of the Institute of Statistical Mathematics 33 (1981), 255 - 262.

[AGA] R. J. Aiyar, C. L. Gouillier and W. Albers, Asymptotic relative efficiencies of rank tests for trend alternatives, Journal of the American Statistical Association 74 (1979), 226 231.

[B] G. K. Bhattacharyya, Tests of randomness against trend or serial correlation, Handbook of Statistics, vol.4. Nonparametric methods (P. R. Krishnaiah and P. K. Sen, eds.), NorthHolland, Amsterdam, 1984, pp. 89 - 111.

[CH] M. Csörgő and L. Horváth, Nonparametric methods for changepoint problems, Handbook of Statistics, vol.7. Quality control and reliability (P. R. Krishnaiah and P. K. Sen, eds.), North-Holland, Amsterdam, 1988, pp. $403-426$.

[CO] A. D. Cliff and J. K. Ord, Spatial processes: Models and applications, Pion, London, 1981.

[DW] J. Durbin and G. S. Watson, Testing for serial correlation in least squares regression I, Biometrika 37 (1950), 409 - 428.

[G] R. C. Geary, The contiguity ratio and statistical mapping, The Incorporated Statistitian 5 (1954), 115 - 145.

[K] A. I. Katsevich, Kernel estimation of the singularities of a signal, Panamerican mathematical journal 5 (1995), no. 1, 1 - 11.

[KO] M. Kendall and J. K. Ord, Time series, 3rd ed., Edward Arnold, UK, 1990. 
[KM] P. R. Krishnaiah and B. Q. Miao, Review about estimation of change points, Handbook of Statistics, vol.7. Quality control and reliability (P. R. Krishnaiah and P. K. Sen, eds.), North-Holland, Amsterdam, 1988, pp. 375 - 402.

[KR1] A. I. Katsevich and A. G. Ramm, Multidimensional algorithm for finding discontinuities of signals from noisy discrete data, Mathl. Comput. Modelling 18 (1993), no. 1, 89-107.

[KR2] , Consistency of rank tests against some general alternatives, Mathl. Comput. Modelling 18 (1993), no. 12, 49-55.

[KR3] - Nonparametric estimation of the singularities of a signal from noisy measurements, Proceedings of the AMS 120 (1994), no. 8, 1221-1234.

[KR4] - Mathematical results in signal and image processing, Doklady, Russian Academy of Sciences 339 (1995), no. 1, 11 - 14.

[KS] M. Kendall and A. Stuart, The advanced theory of statistics, vol. 2, 4th ed., Charles Griffin, London, 1979.

[KW] M. H. Kalos and P. A. Whitlock, Monte Carlo methods. Volume I: Basics, Wiley, New York, 1986.

[L] F. Lombard, Rank tests for changepoint problems, Biometrika 74 (1987), 615 - 624.

[M] E. B. Manoukian, Mathematical Nonparametric Statistics, Gordon and Breach, New York, 1986.

[N] G. E. Noether, Asymptotic properties of the Wald-Wolfowitz test of randomness, Annals of Mathematical Statistics 21 (1950), 231 - 246.

[P] W. K. Pratt, Digital Image Processing, 2nd ed. Wiley, New York, 1991.

[R] A. Renyi, Probability theory, North-Holland, Amsterdam, 1970.

[RK] A. Rosenfeld and A. C. Kak, Digital Picture Processing, Vol. 2, 2nd ed. Academic Press, New York, 1982.

[S] A. Sard, The measure of critical values of differentiable maps, Bull. Amer. Math. Soc. 48 (1942), $883-890$.

[WW] A. Wald and J. Wolfowitz, An exact test for randomness in the nonparametric case based on serial correlation, Annals of Mathematical Statistics 14 (1943), 378 - 388.

Los Alamos National Laboratory, MS B265, CiC-3 Division, Los Alamos, New MeXico 87545

Current address: Mathematics Department, University of Central Florida, Orlando, FL 328161364; e-mail: akatsevi@pegasus.cc.ucf.edu

E-mail address: katsev@c3serve.c3.lanl.gov

Mathematics Department, Kansas State University, Manhattan, KS 66506-2602

E-mail address: ramm@math.ksu.edu 